\title{
LA ESTRUCTURA FINANCIERA DE LAS GRANDES EMPRESAS MANUFACTURERAS MEXICANAS EN EL CONTEXTO DE LA CRISIS FINANCIERA SUBPRIME, 2000-2015
}

THE FINANCIAL STRUCTURE OF LARGE MEXICAN MANUFACTURING ENTERPRISES WHITHIN THE CONTEXT OF THE SUBPRIME CRISIS, 2000-2015

\author{
Francis Magloire Peujio Fozap* \\ Christian Said Domínguez Blancas*
}

enviado: 24 abril 2018 - aceptado: 22 octubre 2018

\begin{abstract}
Resumen
En este trabajo se analizan las repercusiones de la crisis financiera subprime en la estructura financiera de las grandes empresas mexicanas del sector manufacturero en el período 2000-2015. Con base en el marco del planteamiento de la Hipótesis de la Inestabilidad Financiera de Minsky, se estudia la evolución de los estados financieros y un indicador de fragilidad financiera en estas empresas. Los resultados muestran que la crisis acentúo la fragilidad financiera de las grandes empresas manufactureras, la cual se gestó en los años previos como resultado de procesos de inversión en activos físicos e intangibles relativos a la expansión de sus mercados, y que se financiaron mediante deuda de corto y largo plazo. La incidencia de los niveles de endeudamiento en su fragilidad financiera se confirma en la estimación econométrica de datos panel para la muestra de 14 grandes empresas manufactureras analizadas.
\end{abstract}

Clasificación JEL: D21, E12, G33, L6.

Palabras clave: estructura financiera, empresas manufactureras, hipótesis de la inestabilidad financiera.

Peujio Fozap, F., \& Domínguez Blancas, C. (2019). La estructura financiera de las grandes empresas manufactureras mexicanas en el contexto de la crisis financiera subprime, 2000-2015. Estudios económicos, 36 (72), 63-90.

* Escuela Superior de Economía, IPN. Correo electrónico: peufranc@yahoo.fr; cdominguezb@ipn.mx 


\begin{abstract}
This paper analyzes the repercussions of the subprime financial crisis on the financial structure of the large Mexican companies of the manufacturing sector in the period 2000-2015. Based on the framework of the Minsky Financial Instability Hypothesis, we study the evolution of financial statements and an indicator of financial fragility in these companies. The results show that the crisis accentuated the financial fragility of the large manufacturing companies, which was created in the previous years as a result of investment processes in physical and intangible assets related to the expansion of their markets, and which were financed by short and long term debt. The incidence of the levels of indebtedness in its financial fragility is confirmed in the econometric estimation of panel data for the sample of 14 large manufacturing companies analyzed.
\end{abstract}

JEL Code: D21, E12, G33, L6.

Keywords: Financial Structure, Manufacturing Companies, Financial Instability Hypothesis. 


\section{INTRODUCCIÓN}

En la economía mexicana, el sector manufacturero ocupa un papel clave, en tanto es el más importante en términos de la producción bruta total y el tercero en cuanto al personal ocupado (INEGI, 2015), situación que se acentuó, con la apertura comercial y desregulación financiera, y concretamente con la entrada vigor del Tratado de Libre Comercio de América del Norte (TLCAN) en 1994. Estos eventos crearon un punto de inflexión en la economía mexicana, especialmente en las grandes empresas manufactureras, porque implicaron su acceso a nuevos mercados para sus productos y a mercados financieros internacionales. Sin embargo, también las sujetó a su contraparte, la competencia y la inestabilidad financiera internacional. La crisis financiera subprime constituye la evidencia de este último aspecto. Dicha crisis, aunque se originó en el mercado de hipotecas estadounidense, repercutió negativamente alrededor del mundo debido a la alta integración financiera y comercial que caracteriza a la economía actual, y que se refleja en la alineación del movimiento de las variables financieras, especialmente de las tasas de interés (Mántey y Quintana, 2013).

En el caso de la economía mexicana, por su alta integración con la economía estadounidense, la crisis provocó la caída de las exportaciones, el componente más dinámico de la demanda agregada, pero también mayores costos de financiamiento derivados del aumento de las tasas de interés y los tipos de cambio a nivel internacional. De esta manera, se vio afectada la estructura financiera de las grandes empresas manufactureras, es decir, su capacidad de cumplir con sus compromisos financieros, mantener sus actividades y sus procesos de inversión.

Uno de los planteamientos de referencia en el análisis de las estructuras financieras y su evolución durante el ciclo económico es el de Hyman Minsky. En su Hipótesis de la Inestabilidad Financiera (Minsky, 1986), destaca la inherente inestabilidad del sistema económico y cómo, mediante la activa intervención del sistema bancario y sus innovaciones financieras, un sistema caracterizado por una estructura financiera robusta (dominada por empresas cubiertas, que pueden pagar el principal e intereses de la deuda) se transforma en una estructura frágil (empresas especulativas y ponzi, que solo cubren intereses o requieren contratar más deuda para pagar su deudas previas, respectivamente). Minsky sostenía que estos ciclos eran inevitables, empero que el gobierno podía atenuarlos mediante una política monetaria del banco central que guiara las prácticas de los negocios y de financiamiento bancario hacia la creación de estructuras financieras cubiertas. Esto debía ser complementado por una política fiscal con la capacidad de compensar la caída de la inversión privada en la etapa recesiva, pero también de generar superávits 
fiscales, o reducciones del déficit en la etapa ascendente del ciclo, de manera que no se generaran presiones inflacionarias.

Por su poder explicativo, diversos autores han retomado el marco analítico de Minsky (1986) para analizar los efectos de la dinámica actual de los mercados financieros Agosin y Huaita (2011). Y en el caso específico de la crisis subprime de 2008, para estudiar su surgimiento, evolución e implicaciones (Kregel, 2008; Chapoy y Girón, 2009). Con ese propósito, algunos autores construyeron indicadores para estudiar empíricamente las estructuras financieras de empresas y gobiernos durante la crisis. Específicamente, Argitis y Nikolaidi (2014) construyeron dos índices de fragilidad financiera para Grecia (uno aplicable al gobierno de un país no soberano y otro al conjunto de la macroeconomía, i. e., sector público y privado), encontrando que entre 2003-2009, la fragilidad de la economía griega aumentó, situándose en el área que denominaron Ultra-ponzi.

Beshenov y Rozmainsky (2015), por su parte, construyeron un índice o razón de cobertura de intereses (Interest Coverage Ratio) que aplicaron a una muestra de 36 empresas griegas entre 2001 y 2014. Encontraron que la crisis de 2008 provocó que en su mayoría transitaran a estructuras financieras especulativas y ponzi, debido a la caída de sus ganancias y al aumento del costo de refinanciamiento, llevando a la amenaza de bancarrota. Por último, Mulligan, Lirely y Coffee (2014) calcularon una razón de cobertura de intereses (Interest Coverage) para 8707 empresas (extranjeras y domésticas) listadas en mercados estadunidenses durante el período 2002-2009, comprobando que el número de empresas especulativas y ponzi se incrementó a raíz de la crisis subprime, además de reducir su valor de mercado.

Considerando la importancia del sector manufacturero para la economía mexicana, el propósito de este trabajo es analizar los efectos de la crisis financiera subprime en la estructura financiera de las grandes empresas manufactureras. El período de análisis comprende el lapso 2000-2015, con el fin observar comparativamente los cambios que se presentaron en la estructura financiera de estas empresas con el surgimiento de la crisis subprime de 2008.

El análisis de las estructuras financieras es relevante debido a que estas son indicativas del desempeño de la economía, y en especial cuando se trata de sectores clave, como es el caso del sector manufacturero en México. Considerando el marco analítico de la HIF de Minsky, en este trabajo se sostiene como hipótesis que la crisis financiera subprime aumentó la fragilidad financiera de las grandes empresas mexicanas del sector manufacturero, como resultado de la reducción de sus flujos de ingresos no financieros (i. e., ventas) y el aumento del costo de las deudas, 
ambos consecuencia del descenso de la actividad económica estadounidense y del incremento de las tasas de interés y el tipo de cambio.

Es importante advertir que el planteamiento de Minsky se contextualiza en una economía industrializada con un sistema financiero desarrollado, mientras que en el presente caso se analiza una economía en desarrollo con restricción externa de la balanza de pagos.

La particularidad del presente trabajo radica en que se aplica el marco analítico de Minsky al estudio de empresas que integran un sector económico clave en economías en desarrollo abiertas, y donde los cambios en las condiciones comerciales y financieras globales tienen efectos importantes. En este sentido, el presente trabajo va en el propósito de aportar conocimientos que sirvan de base para la elaboración de medidas que permitan atenuar el impacto de los ciclos económicos.

Por último, se advierte que el propósito del trabajo no es proveer una explicación de la crisis financiera subprime, sino sus efectos en la estructura financiera en las grandes empresas manufactureras mexicanas.

El trabajo se divide en cinco partes. Después de esta introducción se realiza una exposición de la Hipótesis de la Inestabilidad Financiera de Minsky, destacando la relación entre el proceso de financiamiento y la evolución de una economía robusta a una frágil. En la tercera parte, se calculan dos índices o razones de cobertura de intereses para una muestra de 14 grandes empresas manufactureras mexicanas. Con base en los resultados, se las clasifica conforme a la taxonomía propuesta por Minsky y se analizan los cambios en su estructura financiera a raíz de la crisis subprime. En la cuarta parte, se estima un modelo econométrico de datos en panel, con la finalidad de determinar las variables financieras con mayor incidencia en el índice o razón de cobertura de intereses. Por último, se presentan las conclusiones.

\section{LA HIPÓTESIS DE LA INESTABILIDAD FINANCIERA (HIF) DE MINSKY}

Minsky (1986) plantea que la economía es inherentemente inestable, sujeta a ciclos de expansión y recesión, cuya génesis está en el curso normal de los negocios que lleva a cambios en las condiciones de financiamiento ${ }^{1}$. En la parte baja

1 Minsky (1986, p. 171) señala que la política económica puede incidir en esta tendencia a la inestabilidad afectando el proceso económico, los salarios y el déficit gubernamental, pero dentro de un entorno capitalista, la inestabilidad no puede ser erradicada totalmente. 
del ciclo estas se caracterizan por el relajamiento del riesgo del prestamista y prestatario, y junto con las innovaciones financieras bancarias, llevan a un incremento de los créditos a una tasa de interés relativamente baja. Esta situación incentiva un mayor endeudamiento de los agentes económicos, transformando una economía robusta en una economía frágil, en donde eventualmente tiene lugar un aumento de los márgenes de seguridad de los bancos y las tasas de interés, y la reducción de los rendimientos actuales. Estos últimos tienen gran importancia, pues su función consiste en validar las inversiones pasadas, por lo que, de no suceder, provocan la disminución del precio de demanda de la inversión (concepto que Minsky retoma de Keynes, 1936) y de los incentivos para invertir.

Para Minsky (1986) la estructura financiera es un elemento central para entender el efecto de los cambios en las condiciones de financiamiento. Según este aspecto, clasifica a las empresas en tres tipos: cubiertas, especulativas y ponzi. En las unidades económicas cubiertas (hedge), los flujos de efectivo generados por la operación normal en el mercado de bienes y servicios son superiores a los pagos de deuda en todos los períodos que dura la inversión. Sus niveles de endeudamiento no son elevados y, por tanto, su estructura financiera no es afectada seriamente por las variaciones en la tasa de interés; sus obligaciones aumentan en montos pequeños y disminuyen el valor presente de la empresa en la misma magnitud, sin ponerla en peligro.

Por su parte, las empresas especulativas se distinguen porque sus flujos de efectivo son superiores, en promedio, a los pagos por deuda, con excepción de los primeros períodos del proceso de inversión, donde los flujos de efectivo son insuficientes para cubrir la parte correspondiente al principal de la deuda, pagando solo los intereses. Por ello, requieren financiamiento adicional y/o de la renegociación de la deuda en las condiciones prevalecientes de la tasa de interés y la disponibilidad crediticia. Por último, las empresas ponzi se distinguen porque en los períodos iniciales sus flujos de efectivo son insuficientes para cubrir el monto del principal y los intereses, debiendo pedir prestado para cubrir estos factores. Las empresas tipo ponzi son las más vulnerables a las condiciones de los mercados financieros debido a la necesidad de renegociar un mayor monto de deuda en los períodos iniciales.

Minsky (1986) argumenta que el tránsito hacia una estructura financiera frágil se explica porque en el inicio del auge los rendimientos de las inversiones son altos y la tasa de interés es baja, pero esto se modifica a medida que el ciclo avanza y las decisiones de inversión se incrementan por encima de la capacidad de finan- 
ciamiento interno de las empresas, dada por sus utilidades corrientes ${ }^{2}$. Empero, sus altos rendimientos permiten incrementar su capacidad de endeudamiento.

Los bancos desempeñan un papel decisivo en esta situación, puesto que con el fin de incrementar sus ganancias inducen a una mayor inversión financiada con deuda. La particularidad es que los bancos avalan las decisiones de inversión proporcionando el financiamiento adicional a través de innovaciones financieras que permiten aumentar los medios de pago sin elevar la tasa de interés.

La consecuencia del mayor nivel de apalancamiento es que en períodos posteriores las empresas deberán destinar mayores flujos financieros al pago de la deuda, lo cual puede reducir el gasto de inversión, así como los futuros rendimientos, a tal punto que no se cubran los compromisos financieros. En otras palabras, la estructura financiera se vuelve más frágil, porque aumenta la necesidad de refinanciamiento de la deuda, independientemente del incremento de la tasa de interés.

Con base en lo anterior, Minsky (1986) advierte que una economía donde predominan empresas con una estructura financiera cubierta no está libre de evolucionar hacia una estructura financiera frágil (dominada por empresas especulativas y ponzi). En ella, puede observarse el incumplimiento de los compromisos y la bancarrota de las empresas ponzi, lo cual afecta negativamente los ingresos de otros agentes económicos, impidiéndoles también validar sus deudas.

La crisis del sistema puede desencadenarse cuando los bancos perciben que el nivel de endeudamiento está por encima de lo que consideran normal, modificando las condiciones de financiamiento. Específicamente, en la medida en que aumenta el financiamiento externo se reducen los márgenes de seguridad y aumentan los riesgos del prestamista y prestatario. Como consecuencia, el prestamista restringe las condiciones de financiamiento (i. e. aumenta la tasa de interés), incidiendo en las decisiones de inversión y en el curso de las inversiones corrientes.

Adicionalmente, la implementación de políticas monetarias restrictivas, en un intento por detener las presiones inflacionarias provocadas por la expansión inicial de la oferta monetaria realizada por los bancos, puede reforzar la postura de los bancos comerciales. Sin embargo, ello fortalece el encarecimiento y la reducción de la disponibilidad de financiamiento.

2 Minsky (1986) también coincide con Keynes (1936) en que en estas condiciones el precio de demanda de la inversión, o expectativa de los rendimientos futuros de los bienes de capital, es superior al precio de oferta. Ello se traduce en el aumento de las decisiones de inversión. 
Minsky sostiene que al final el incremento en las tasas de interés impone mayores cargas financieras a las empresas, disminuye el precio de demanda de la inversión e incrementa el precio de oferta; además, pone en riesgo la conclusión de las inversiones en marcha y la realización de nuevas inversiones ${ }^{3}$. La necesidad de obtener recursos líquidos para concluir las inversiones y/o para pagar las deudas provoca el aumento de la oferta de bonos de empresas y bancos, quienes compiten por vender sus activos financieros, generando un proceso de deflación en el precio de los activos que incrementa aún más la iliquidez en el sistema ${ }^{4}$. Aumenta el incumplimiento de los compromisos financieros y tiene lugar una crisis que deprime aún más la inversión, el empleo y el ingreso de la economía.

Resumiendo, la particularidad del planteamiento de Minsky consiste en sostener que la economía está inherentemente sujeta a ciclos de expansión y recesión, con origen en el aumento de las necesidades de financiamiento avaladas por el sistema bancario. Sin embargo, las condiciones de financiamiento y la tasa de interés se modifican con el ciclo, induciendo una estructura financiera frágil, caracterizada por el predominio de empresas de tipo especulativas y/o ponzi, cuya vulnerabilidad es mayor ante variaciones en la tasa de interés.

En el caso particular de las economías en desarrollo, trabajos como el de Agosin y Huaita (2011) destacan la vulnerabilidad que experimentan estas economías como consecuencia de la entrada y salida de capitales, y plantean la necesidad de contar con políticas específicas para atenuar los efectos de estos procesos endógenos de los mercados financieros. Al respecto puede señalarse que la economía mexicana es un caso representativo, pues no solo se encuentra sujeta a las oleadas de capital, sino también a la inestabilidad proveniente del descenso en la actividad de su principal socio comercial.

Considerando el marco analítico de Minsky, a continuación, se revisa la evolución de la estructura financiera de las grandes empresas manufactureras de México en el contexto de la crisis subprime.

3 Adicionalmente, el precio de demanda puede ser afectado si la compra de los bonos y acciones se realizó con financiamiento bancario de corto plazo (Minsky, 1986, p. 195).

4 Minsky (1986, Cap. 9) señala que las empresas cubiertas y, en mayor grado las especulativas y ponzi, por su mayor dependencia a las variaciones de la tasa de interés, mantienen dinero en efectivo y/o activos financieros para diversificar su portafolio y, así poder solventar el pago de sus deudas, en caso de que se interrumpan los flujos de efectivo o la disponibilidad de financiamiento en condiciones favorables. 


\section{EVOLUCIÓN DE LA ESTRUCTURA FINANCIERA DE LAS GRANDES EMPRESAS MANUFACTURERAS MEXICANAS, 2000-2015}

El desempeño de la economía mexicana desde la década de los noventa, especialmente a partir de la entrada en vigor del Tratado de Libre Comercio de América del Norte en 1994, se caracteriza porque las exportaciones adquieren mayor relevancia en la dinámica del crecimiento económico (Palley, 2012). Sin embargo, una de las debilidades del modelo basado en las exportaciones es que México realiza más del $80 \%$ de su intercambio comercial con Estados Unidos de América (Cuevas, 2013). Esto ha otorgado a la economía mexicana un carácter cíclico, en términos del PIB (Garrido y Ortiz, 2009), y subordinado a la dinámica de la economía estadounidense.

En este contexto, la crisis financiera subprime, aunque no se originó en la economía mexicana, ni su principal mecanismo de transmisión fue a través de los activos financieros hipotecarios estadounidenses, sí tuvo efectos importantes en el sector productivo. Esto se explica por esa alta integración comercial con la economía estadounidense. Así, a partir del tercer trimestre de 2008 se inició la disminución en las exportaciones, con una caída de 1,5\% respecto del mismo trimestre del año anterior, situación que se acentuó en el último trimestre, cuando ascendió a 9,42\%. En 2009 la situación empeoró y la demanda externa continuó disminuyendo, por lo que las exportaciones mexicanas se redujeron $20,27 \%$ durante el primer semestre de $2009^{5}$. Al final del año las exportaciones mexicanas reflejaron una disminución anual del 13,7\% (Banco de México, 2010).

La crisis también afectó otras variables relevantes para la economía mexicana como las remesas, la Inversión Extranjera Directa (IED), la demanda de crudo, las restricciones crediticias, y los flujos de capital (Cuevas, 2013). Como resultado, el PIB creció solo 1,8\% en 2008, y tuvo una fuerte caída de 6,1\% en 2009 (Banco de México, 2010).

Las grandes empresas del sector manufacturero, con una parte de su actividad orientada al exterior, fueron especialmente afectadas. En particular, la hipótesis es que la crisis provocó la reducción de sus flujos de efectivo y aumentó sus obligaciones, y de esta manera incrementó su fragilidad financiera. Con el objetivo de aportar evidencia relativa a este argumento, a continuación se analiza la evolución de los activos, ingresos, pasivos y gastos de una muestra de grandes empresas

5 www.banxico.org.mx/SieInternet/consultarDirectorioInternetAction.do?sector=2\&accion=consult arCuadro\&idCuadro $=\mathrm{CR} 151 \&$ locale $=\mathrm{es}$ 
manufactureras mexicanas. Posteriormente, se determina el grado de fragilidad del conjunto de empresas en el marco de la clasificación propuesta por Minsky. Esto se realiza a partir del cálculo de los índices o razones financieras propuestas por Mulligan, Lirely y Coffee (2014), y Beshenov y Rozmainsky (2015). El análisis comprende el período 2000-2015, con el propósito de distinguir los cambios en la estructura financiera de las empresas provocados por la crisis subprime.

Para fines del presente trabajo la muestra está integrada por 14 grandes empresas manufactureras con matriz en México y listadas en la Bolsa Mexicana de Valores (BMV), las cuales tienen acceso a los mercados financieros internacionales y las acciones representativas de su propiedad pueden ser adquiridas por inversionistas extranjeros, parcial o totalmente, y que cuentan con la información financiera requerida para el período de análisis (véase tabla 1).

Tabla 1. Muestra de grandes empresas del sector manufacturero

\begin{tabular}{ll}
\hline \multicolumn{1}{c}{ Empresa } & \multicolumn{1}{c}{ Actividades } \\
\hline Alfa & Fabricación de productos metálicos forjados y troquelados. \\
\hline Bio Pappel & Fabricación de celulosa, papel y cartón. \\
\hline Cementos Chihuahua & Fabricación de cemento y productos de concreto. \\
\hline Cemex & Fabricación de cemento y productos de concreto. \\
\hline Convertidora Industrial & Fabricación de productos de plástico. \\
\hline Cydsa & Fabricación de otros productos químicos. \\
\hline Grupo Industrial Saltillo & $\begin{array}{l}\text { Fabricación de productos a base de arcillas y minerales } \\
\text { refractarios. }\end{array}$ \\
\hline Grupo Kuo & $\begin{array}{l}\text { Fabricación de otra maquinaria y equipo para la industria } \\
\text { en general. }\end{array}$ \\
\hline Grupo Pochteca & Fabricación de productos químicos básicos. \\
\hline Hylsamex & Fabricación de productos de hierro y acero. \\
\hline Inter Cerámica & $\begin{array}{l}\text { Fabricación de productos a base de arcillas y minerales } \\
\text { refractarios. }\end{array}$ \\
\hline Lamosa Gpo. & $\begin{array}{l}\text { Fabricación de productos a base de arcillas y minerales } \\
\text { refractarios. }\end{array}$ \\
\hline Mexichem & Fabricación de otros productos químicos. \\
\hline Vasconia & Fabricación de productos metálicos forjados y troquelados. \\
\hline Fin.....
\end{tabular}

Fuente: elaboración propia con base en datos de Economatica. 
Las estadísticas que se utilizan corresponden a diversos rubros de sus estados financieros (balance general y estado de resultados) obtenidos de la base de datos Economatica. En el caso del balance, se usan cifras al último trimestre de cada año, mientras que en el caso de los rubros del estado de resultados se ocupan flujos anuales y expresados en millones de pesos constantes al 31 de diciembre de 2015.

En primer lugar, se muestra la evolución de los activos con el propósito de determinar el destino de los recursos de las grandes empresas manufactureras. Se observa que sus activos totales tuvieron un comportamiento creciente en el período de estudio, aunque se distinguen dos etapas. La primera de 2000 a 2008, en la que tienen una pendiente más pronunciada, que denota un crecimiento mayor en un período de relativa estabilidad económica internacional, con excepción de la recesión de 2001. La segunda etapa va de 2010 a 2015, y se inició después de dos caídas consecutivas en 2009 y 2010 , que recogen el efecto de la crisis subprime, provocando un relativo estancamiento de los activos hasta 2013 (gráfico 1).

Gráfico 1. Principales rubros del activo y pasivo de las grandes empresas manufactureras, 2000-2015 (\% del activo total)

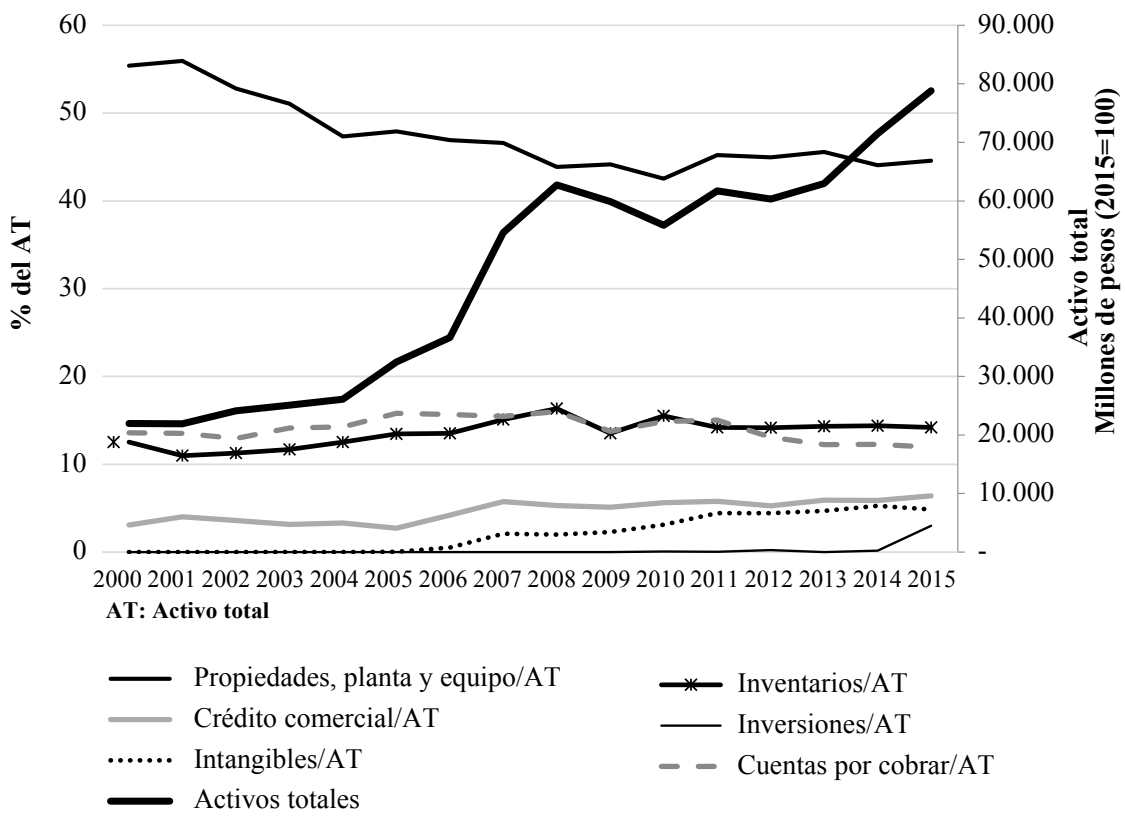

Fuente: elaboración propia con base en datos de Economática. 
En cuanto a la composición de los activos, se observa, como podría esperarse en empresas manufactureras, que su principal rubro son las propiedades, planta y equipo (PPE), que agrupa a los activos físicos utilizados en la producción y distribución de mercancías. Este concepto representó en promedio el 47,4\% de los activos totales, aunque se percibió una tendencia decreciente de su participación en los activos totales, del 55,4\% en el año 2000 al 44,6\% en 2015 (gráfico 1) 6.

Asimismo, llama la atención que la crisis tuvo un efecto retardado en las inversiones físicas de las empresas manufactureras, en tanto fue hasta 2010 cuando se apreció la disminución de la participación de las PPE en los activos totales. Esto se explica porque las inversiones implican altos costos hundidos, por lo que una vez iniciadas en ocasiones resulta menos costoso concluirlas que cancelarlas.

Las cuentas por cobrar son otro rubro del activo que incluye las deudas a cargo de clientes y los anticipos realizados para la obtención de bienes y servicios, entre otros conceptos. Estas tuvieron un comportamiento relativamente estable, con una participación promedio del $14 \%$ respecto de los activos totales en el período de estudio. Destaca que en 2008 tuvieron su mayor participación, del 16\% del activo total, reflejo del aumento de las ventas a crédito debido los problemas de liquidez que presentaron sus clientes (gráfico 1).

Los inventarios son otro rubro importante de las empresas manufactureras, representativo de las mercancías terminadas e insumos. Su tendencia ascendente entre el 2000 y 2008 fue reflejo de una mayor actividad, que se interrumpió en 2009. Después de ese año, mantuvieron una participación de alrededor del 14\% de los activos totales, sin alcanzar nuevamente el máximo de 16\% de 2008.

Otra parte de los activos son los denominados intangibles, que se subdividen en dos rubros. En primer lugar, el crédito comercial, el cual refleja el valor contable que se le asigna al nombre de la empresa y las relaciones con los clientes. Este rubro representó en promedio el 4,7\% del activo total, y su rasgo más significativo fue que duplicó su participación, al pasar del 3,1\% en 2000 al 6,4\% de los activos totales en 2015.

Un comportamiento similar tuvo el resto de los intangibles (i. e., intangibles diferentes al crédito comercial), cuya participación en los activos totales pasó de

6 Una parte de la literatura asocia esta política al denominado proceso de financiarización que en las corporaciones no financieras de países desarrollados se refleja en la adopción del principio de "reducir y redistribuir", es decir, reducir las inversiones y la planta laboral e incrementar la distribución de los dividendos (Stockhammer, 2004). 
prácticamente cero en 2000 al 4,9\% en 2015. Esta segunda clase de intangibles refleja las inversiones en rubros como patentes y marcas, propias u obtenidas en procesos adquisiciones y fusiones con otras empresas. Su característica es que producen ventajas industriales o poder de mercado frente a competidores, y por este motivo se traducen en la posibilidad de incrementar los ingresos y el valor de la empresa.

La importancia de los intangibles en los activos de las empresas es un rasgo representativo del capitalismo contemporáneo de aparición relativamente reciente en las empresas mexicanas. En el caso de estas últimas, dada su baja capacidad de innovación (Garrido y Ortiz, 2009), reflejan principalmente activos adquiridos como consecuencia de procesos de fusión y adquisición para expandirse hacia el resto de América Latina y Estados Unidos?.

Por último, el rubro de inversiones financieras destaca por su reciente aparición en 2010, que coincide con la caída de la actividad de las empresas derivadas de la crisis subprime, y sugiere que canalizaron recursos a actividades financieras para compensar su caída de ingresos en su principal área de negocios. No obstante, su participación en los activos totales solo alcanzó el 3\% en 2015.

Por otro lado, respecto al comportamiento de los pasivos, se observa que aquellos de largo plazo son los de mayor importancia en las grandes empresas manufactureras. Representaron en promedio el $28,2 \%$ de los pasivos totales, y se distinguen tres etapas en su comportamiento. En la primera de 2000 a 2006, mantuvieron una participación de alrededor del $31 \%$ de los activos totales, en un contexto de tendencia creciente en los pasivos totales (gráfico 2). Dicho comportamiento estuvo vinculado al financiamiento de las inversiones físicas y a la adquisición de activos intangibles.

La segunda etapa corresponde a los años 2007 y 2008, en los que se observó su participación máxima y mínima, que se explican por el aumento de las variables financieras (tasa de interés y tipo de cambio) y la menor disponibilidad de financiamiento, respectivamente. Después de la crisis, entre 2009 y 2013, los pasivos de largo plazo decrecieron, con un relativo incremento en los dos últimos años.

Por su parte, las deudas de corto plazo, relacionadas a cubrir compromisos inmediatos de las empresas ( $v . g$., capital de trabajo), representaron una menor proporción del pasivo de las grandes empresas manufactureras, $10,1 \%$, en promedio, de los pasivos

7 Véase Garrido (2005) para una discusión de los procesos de expansión realizados por las grandes empresas mexicanas. 
totales. Su rasgo más notable fue la tendencia descendente que tuvieron entre 2000 y 2007, a lo que siguió su nivel máximo en 2008 (23,1\% de los activos totales), que estuvo vinculado a la recontratación de deudas a tasas de interés más altas tanto en el interior como en el exterior (gráfico 2) ${ }^{8}$, además del aumento de las deudas denominadas en moneda extranjera, que debieron pagarse a un tipo de cambio mayor. Este último pasó de un promedio de 10,9 pesos por dólar en 2007 a 11,1 y 13,5 pesos por dólar en 2008 y 2009, respectivamente. A partir de 2009, los pasivos de corto plazo se redujeron, pero se mantuvieron por encima de los niveles observados entre 2004 y 2007.

Por último, los proveedores que representan los financiamientos recibidos por las empresas de los vendedores de insumos no experimentaron grandes cambios durante el período de estudio.

Gráfico 2. Principales rubros del pasivo de las grandes empresas manufactureras, 2000-2015 (\% del pasivo total)

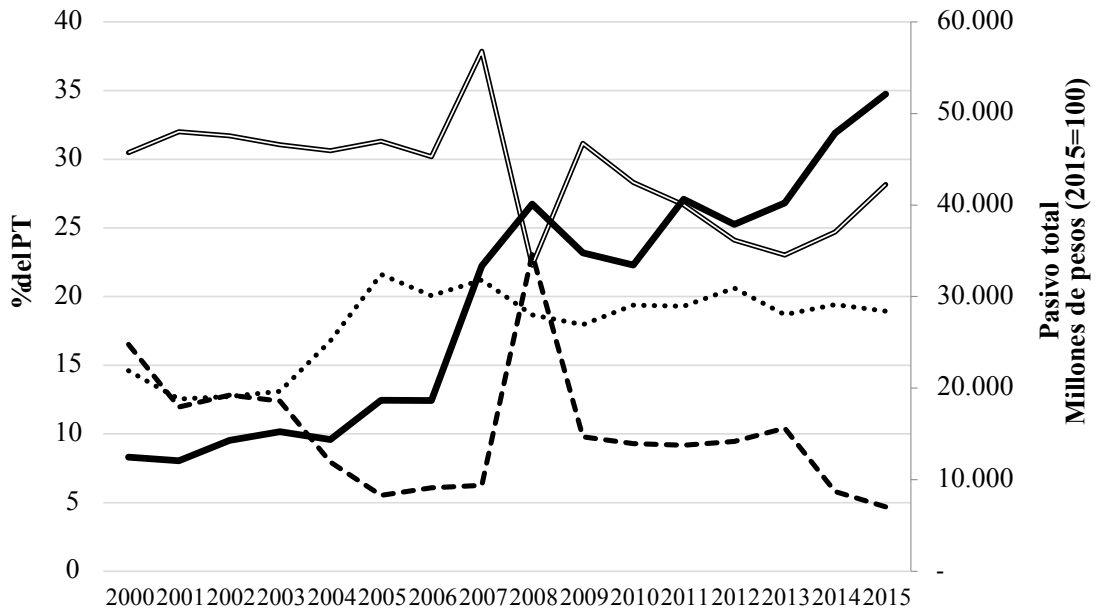

PT: Pasivo total

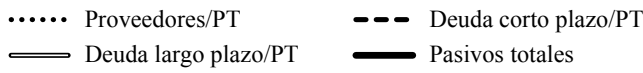

Fuente: elaboración propia con base en datos de Economática.

8 Véase Mántey y Quintana (2013), quienes aportan evidencia sobre la integración de los mercados financieros y su reflejo en las tasas de interés. 
Por otra parte, los ingresos indican la eficacia con que las empresas generan ganancias. Para su análisis, se retoma la clasificación de la fuente estadística, en ingresos financieros y no financieros. Los primeros incluyen intereses y ganancias cambiarias, mientras que los segundos comprenden principalmente ingresos provenientes por la venta de los bienes manufacturados, así como otros complementarios a sus actividades.

Los ingresos no financieros, por un amplio margen, son los más importantes de las grandes empresas manufactureras. Representaron en promedio el 99\% del total de ingresos durante el período de estudio. Tuvieron una tendencia creciente, con excepción de una relativa caída en 2009, asociada a la disminución de sus ventas.

Gráfico 3. Ingresos y gastos de las grandes empresas manufactureras, 2000-2015 (millones de pesos, $2015=100$ )

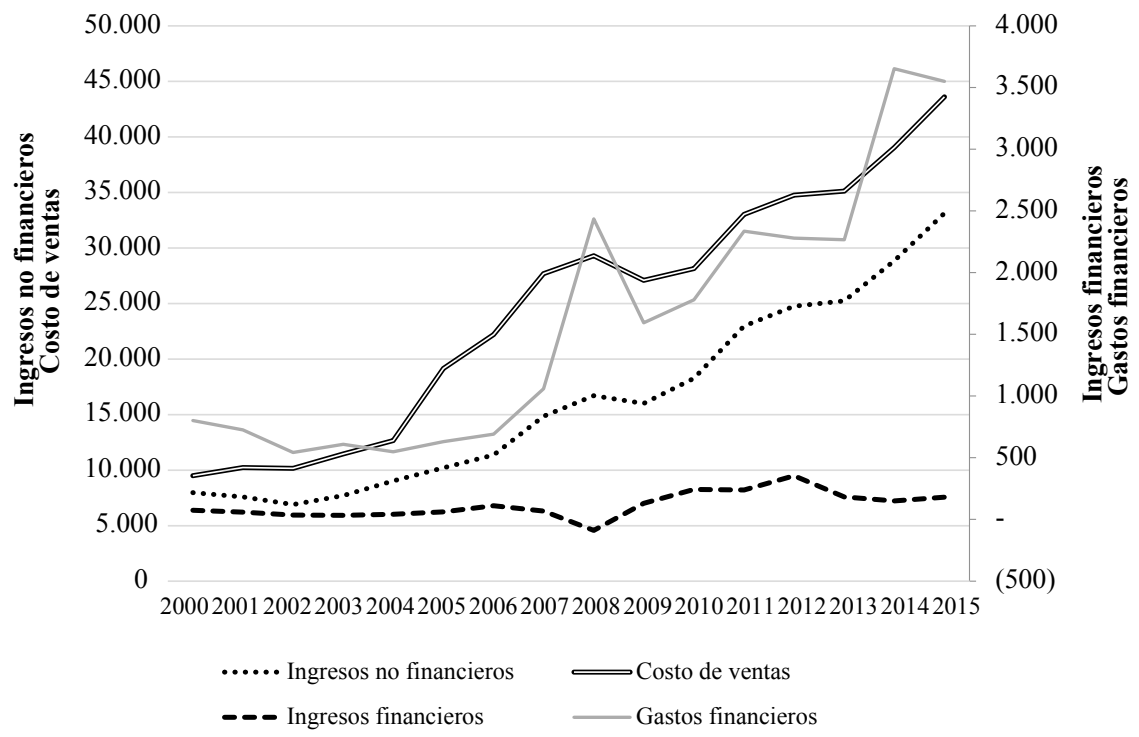

Fuente: elaboración propia con base en datos de Economática.

En cuanto a los ingresos financieros, se observa que estos representan una proporción mucho menor, en promedio el $1 \%$ de los ingresos totales. Destaca que fueron negativos en 2008, reflejando el efecto inmediato de la crisis en las variables financieras como las tasas de interés y el tipo de cambio. Entre 2009 y 2012 , tuvieron su participación más alta, lo cual se asoció también a la aparición de las inversiones financieras (gráfico 3). 
Por otro lado, el costo de ventas, su desembolso operativo más importante, creció de manera constante durante todo el periodo de estudio, en correspondencia con la evolución de sus ingresos no financieros (gráfico 3). Su mayor incremento en el 2008 fue consecuencia del mayor precio de los insumos, particularmente aquellos de origen importado.

Por su parte, los gastos financieros se incrementaron entre 2006 y 2008, particularmente en este último año, manteniéndose en estos niveles hasta 2013. En el resto del período experimentaron aumentos moderados.

Es importante mencionar que, aunque los gastos financieros representan una menor proporción de los gastos de las grandes empresas, en períodos de inestabilidad financiera experimentan considerables incrementos que pueden tener efectos significativos en las ganancias. Por ejemplo, en términos del costo de ventas representaron una vigésima sexta parte en 2007, pero en 2008 representaron la doceava parte. Esta situación también se observó en otros períodos de incertidumbre económica como 2000 y 2014, donde representaron alrededor de una doceava y onceava parte del costo de ventas, respectivamente.

Resumiendo, los resultados indican que, en los años previos a la crisis de 2008, las grandes empresas manufacturera realizaron procesos de inversión en activos, especialmente en los activos intangibles derivados de su expansión al mercado estadounidense (en el marco del TLCAN) y, en menor intensidad, a otros mercados como el europeo y latinoamericano. Empero, debido a que las grandes empresas manufactureras financiaron sus inversiones con pasivos de corto y largo plazo, la emergencia de la crisis incrementó sus compromisos financieros. A ello se agregó la reducción de sus ingresos por ventas. En términos de Minsky, la crisis impidió validar sus inversiones pasadas, reflejándose en el estancamiento de sus procesos de inversión.

Para determinar el efecto de la crisis en la capacidad de las empresas para cubrir sus compromisos financieros, se retoman las metodologías propuestas por Mulligan, Lirely y Coffee (2014), y Beshenov y Rozmainsky (2011) para el cálculo de dos indicadores de fragilidad financiera. Con base en los resultados se evalúa el grado en que aumentó la fragilidad financiera del conjunto de grandes empresas del sector manufacturero.

La primera metodología es la propuesta por Mulligan, Lirely y Coffee (2014), los cuales calculan una razón de cobertura financiera, IC (siglas en inglés de Interest Coverage), definida de siguiente manera: 


$$
I C=\frac{\text { ingreso } \text { neto }+ \text { gastos por intereses }}{\text { gastos por intereses }}
$$

Esta razón financiera evalúa la capacidad de las empresas para cubrir sus gastos financieros a partir de su ingreso neto 9 . Si el valor del IC es mayor o igual a 4, es considerada una empresa cubierta. Si está entre 0 y 4 la empresa es especulativa. Pero si es menor a cero, la empresa tiene una estructura ponzi, lo que indicaría que ingresos son inferiores a sus compromisos financieros.

El comportamiento del IC promedio del conjunto de empresas manufactureras indica que entre 2001 y 2006 mejoró la cobertura de sus compromisos financieros, pero que se deterioró en 2007, y especialmente en 2008, denotando los efectos de la crisis subprime en la capacidad de las grandes manufactureras para cumplir con sus compromisos financieros (gráfico 4a). Esto es, la fragilidad del conjunto de empresas se incrementó con la crisis subprime, lo que se asoció al aumento de los pasivos y de insumos como consecuencia del aumento de las tasas de interés y la depreciación cambiara.

Posteriormente, entre 2009 y 2015, se apreció una nueva disminución de la capacidad de cobertura de las grandes empresas, dado que el IC pasó de 4,2 en 2009 a 2,8 en 2015; la excepción fue 2012, en el cual, el IC alcanzó un valor cercano a 12, debido a un nivel extraordinario del IC de Grupo Industrial Saltillo que incidió en el promedio de la muestra.A nivel individual, se observa que, con excepción de tres empresas (Cementos Chihuahua, Grupo Industrial Saltillo y Vasconia), la mayoría tuvo un IC promedio menor a 4, es decir, reflejaron una estructura financiera de tipo especulativa, y, en consecuencia, fueron más susceptibles de ser afectadas por cambios repentinos en las condiciones financieras (gráfico $4 \mathrm{~b}$ ).

A partir de los resultados anteriores, puede señalarse que la crisis profundizó la fragilidad financiera de las empresas manufactureras. Como se señaló, ello estuvo asociado al aumento de sus inversiones en activos, especialmente los intangibles, cuya adquisición fue financiada mediante deuda de corto y largo plazo, en un entorno de alta inestabilidad financiera que caracteriza a los mercados financieros.

9 El ingreso neto está definido como la ganancia o pérdida neta, es decir, los ingresos después de intereses e impuestos. 
Gráfico 4a. Comportamiento del IC promedio del conjunto de grandes empresas manufactureras, 2000-2015

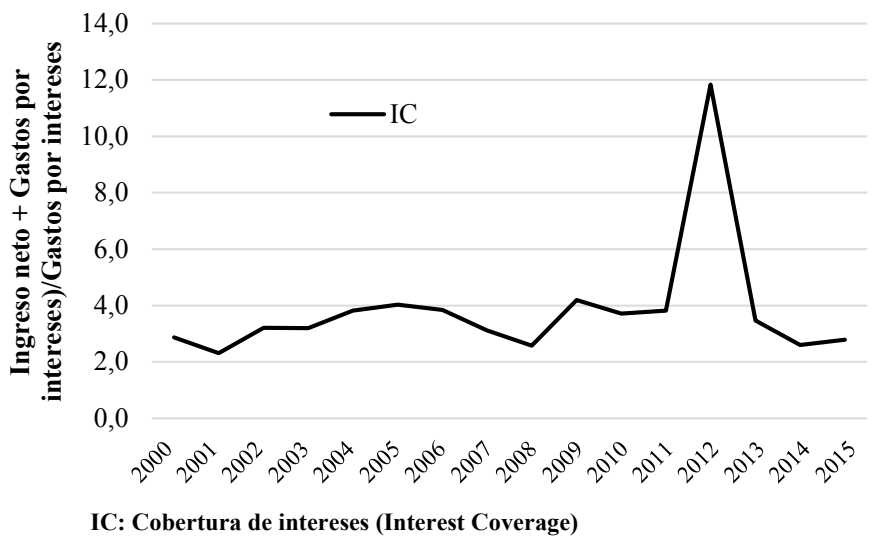

Fuente: elaboración propia con datos de Economática.

Gráfico 4b. Comportamiento del IC promedio individual de las grandes empresas manufactureras, 2000-2015

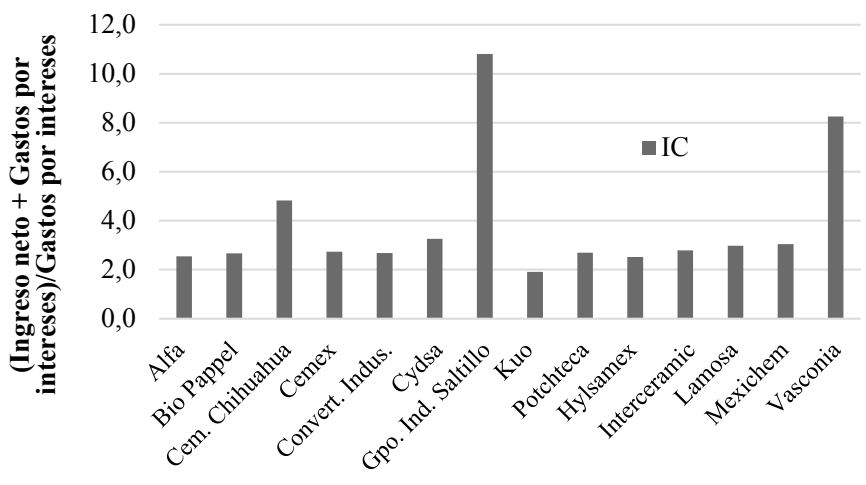

IC: Cobertura de intereses (Interest Coverage)

Fuente: elaboración propia con datos de Economática. 
Razón de cobertura de la tasa de interés: IRC

Por otra parte, Beshenov y Rozmainsky (2015) propusieron la razón de cobertura de intereses, IRC (siglas en inglés de Interest Coverage Ratio) para evaluar el grado de fragilidad financiera. Dicha razón es definida de la siguiente forma:

$$
I R C=\frac{E B I T}{\text { Gastos por intereses }}
$$

Donde el EBIT representa las ganancias antes de intereses e impuestos (Earnings Before Interest and Taxes)

Basándose en los análisis de Damodaran (2011) y Teplova (2011), Beshenov y Rozmainsky (2015) definen que una empresa es financieramente estable, "resistente" a los choques externos, cuando tiene un IRC mayor a 3, es decir, si con las ganancias antes de intereses e impuestos cubre más de tres veces sus compromisos financieros. Si el IRC está entre cero y 3 , la empresa es especulativa. Pero si el IRC es menor a cero, la empresa es considerada ponzi. Esta metodología es más rigurosa que la previa porque en el numerador considera las ganancias antes de impuestos, es decir, los ingresos después de deducir el costo de ventas y los costos administrativos. En el presente caso, para calcular el IRC de forma más rigurosa, en el denominador se consideró la suma de los rubros gastos financieros y otros gastos financieros ${ }^{10}$.

El comportamiento del IRC promedio para el conjunto de empresas se mantuvo por encima de 3 para la mayor parte de los años, es decir, reflejó una estructura financiera cubierta. Sin embargo, con la emergencia de la crisis descendió a 3 en 2007 y a 1,9 en 2008 , indicando que se transitó hacia una estructura financiera predominantemente especulativa (gráfico 5a). En el resto del período de estudio, la muestra de empresas en su conjunto mantuvo una estructura financiera cubierta, en tanto el IRC promedió 4,4; especialmente en 2011 y 2012, explicado por el alto valor del IRC para Vasconia en 2011, y Grupo Industrial Saltillo y Vasconia en 2012.

A un mayor nivel de detalle, la estructura financiera individual de las empresas muestra que la mayoría ( 9 de 14) tuvo, en promedio, una estructura financiera cubierta, en tanto, su IRC fue superior a 3. Sin embargo, en términos porcentuales, equivale a que alrededor del $35,7 \%$ de la muestra tuvo una estructura especulativa (gráfico 5b).

10 En ciertos períodos fue necesario calcular el EBIT = Resultado Bruto - Gastos en ventas - Gastos administrativos + Otros ingresos operacionales - Otros gastos. 
Gráfico 5a. Comportamiento del IRC promedio del con junto de grandes empresas manufactureras, 2000-2015

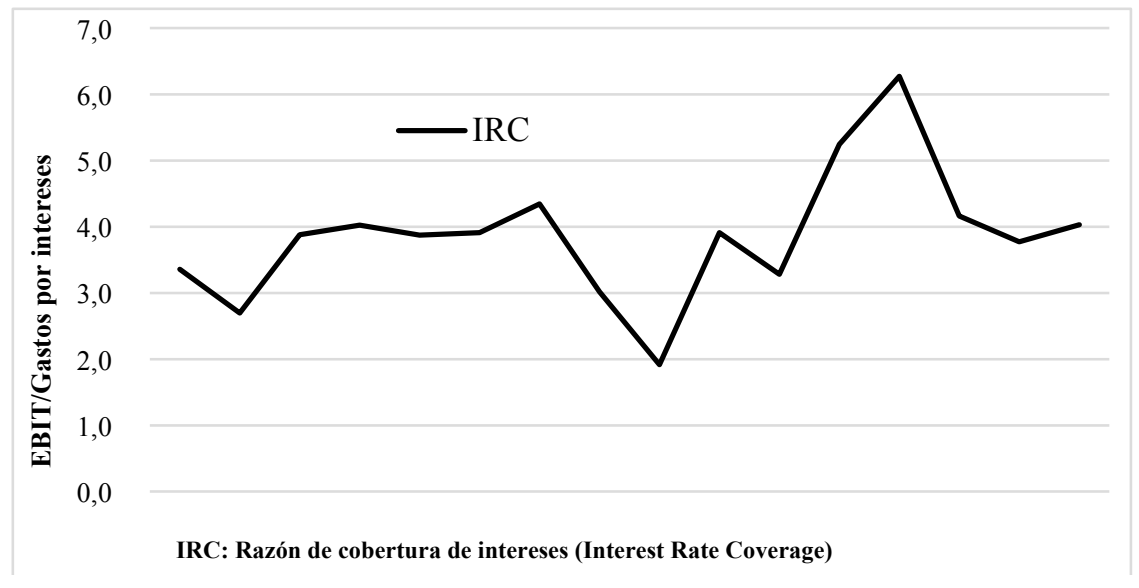

Fuente: elaboración propia con datos de Economática.

Gráfico 5b. Comportamiento del IRC promedio individual de las grandes empresas manufactureras, 2000-2015

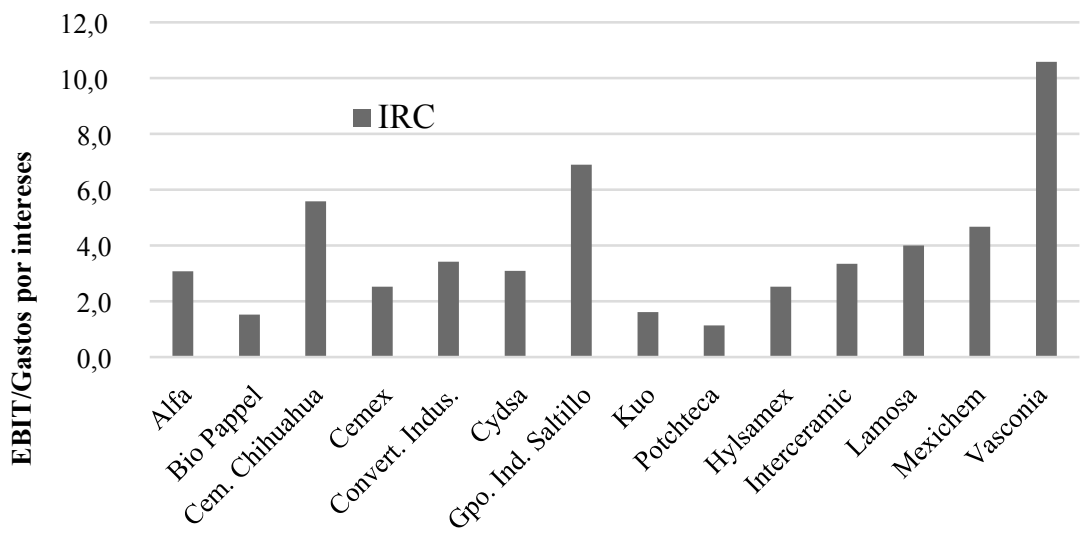

IRC: Razón de cobertura de intereses (Interest Rate Coverage)

Fuente: elaboración propia con datos de Economática. 
Por tanto, a partir de los resultados anteriores, es posible señalar que a raíz de la crisis subprime se acentuó la fragilidad financiera de las grandes empresas manufactureras mexicanas. Sin embargo, también puede decirse que dicha fragilidad se gestó antes de la misma, como resultado de procesos de inversión financiados mediante endeudamiento, y por el bajo crecimiento de la economía en su conjunto.

Por tanto, los resultados avalan lo establecido por Minsky: el financiamiento de expansiones con recursos externos lleva a situaciones de mayor fragilidad financiera ante cambios en las condiciones de financiamiento que se presentan en el ciclo económico. En el caso de la economía mexicana, el aumento de la fragilidad en las empresas manufactureras evidencia también su alta dependencia del desempeño de la economía estadounidense.

\section{ESTIMACIÓN DE LOS PRINCIPALES DETERMINANTES DE LA FRAGILIDAD FINANCIERA DE LAS EMPRESAS MANUFACTURERAS MEXICANAS}

De acuerdo con Minsky, la fragilidad financiera es una situación de carácter cíclico que se caracteriza por un mayor endeudamiento y el aumento de los compromisos financieros por encima de los ingresos.

En las grandes empresas manufactureras mexicanas se corrobora la pertinencia del uso del marco analítico de Minsky, en tanto se observó que estas incrementaron su endeudamiento para financiar sus procesos de inversión y expansión en un contexto de tipos de cambio y tasas de interés relativamente bajos y estables en los mercados financieros internacionales. Sin embargo, estas condiciones se revirtieron con la emergencia de la crisis subprime, originando estructuras financieras más frágiles.

Las causas de la mayor fragilidad financiera, como se argumenta en la hipótesis, pueden encontrarse la reducción de sus flujos de ingresos no financieros (i.e., ventas) y el aumento del costo de las deudas, ambos consecuencia del descenso de la actividad económica estadounidense y del incremento de las tasas de interés y el tipo de cambio, y que afectaron el desempeño de las grandes empresas manufactureras.

Para aportar evidencia que apoye o rechace la hipótesis anterior, en este apartado se realiza una estimación econométrica, con el propósito de determinar las variables que más influyeron en la fragilidad de las grandes empresas manufactureras, medida a través de la razón de cobertura de intereses (IRC). Dado que 
el análisis se realiza para la muestra de 14 empresas del sector manufacturero, se consideró adecuado realizar la estimación utilizando una técnica econométrica para datos panel.

La selección del IRC como variable dependiente, de entre los dos indicadores de fragilidad analizados en el apartado anterior, obedece a que constituye una medida más rigurosa, en tanto supone que los compromisos financieros se cubren a partir de las ganancias antes de impuestos, y después de que se cubren los gastos operativos (lo que correspondería a lo que se conoce como EBIT).

Las variables independientes o explicativas consideradas en la estimación incluyen los rubros más importantes del pasivo, primordialmente las deudas de corto y largo plazo de cada empresa, como proporciones del patrimonio neto. De esta manera, se capta el grado de apalancamiento, y el efecto que tiene la composición del financiamiento entre fuentes internas y externas. Asimismo, se incluyen otras variables de carácter macroeconómico, cuya evolución puede acentuar o atenuar la capacidad de las empresas para cubrir sus compromisos financieros. Entre estas variables se incluyen la tasa de interés, el tipo de cambio, los términos de intercambio, el gasto de gobierno y el consumo de los hogares.

La estimación se realiza para las 14 empresas de la muestra, con datos anuales por el período 2000-2015. Las variables utilizadas en la estimación fueron obtenidas de Economatica, Banco de México, INEGI y de la Reserva Federal.

Para determinar el impacto en el indicador de fragilidad financiera se realizó la estimación de las tres especificaciones estándar en datos en panel, que son datos agrupados, efectos fijos y efectos aleatorios, a partir de la siguiente ecuación:

$$
Y_{i t}=\alpha^{i}+\beta X_{i t}+\varepsilon_{i t} \text { para } \mathrm{i}=1, \ldots, \mathrm{N} \text { and } \mathrm{t}=1, \ldots, \mathrm{T}
$$

Donde $Y$ es la variable dependiente, en este caso el índice de cobertura de los intereses (IRC); $X$ es un vector de variables explicativas, que incluye la razón de la deuda de corto plazo al patrimonio neto , la razón de la deuda de largo plazo al patrimonio neto , el índice de los términos de intercambio medido en dólares (Itidlls), la tasa de crecimiento promedio anual de las exportaciones (X), la tasa de interés interbancaria a 91 días (rMex), la tasa de crecimiento anual del consumo de gobierno (CGC), y la tasa de interés de los fondos federales de los EUA ; es la ordenada al origen; es el vector de coeficientes de las variables explicativas; es el término de error que satisface los supuestos del ruido blanco, representado como $\varepsilon_{i t} \sim$ iid $\left(0, \sigma_{\varepsilon}\right)$, es decir, independiente e idénticamente distribuido con media 0 la varianza constante $\sigma_{\varepsilon}$. 
En primer lugar se estima el modelo de datos agrupados o MCO, el cual supone que, es decir, que el intercepto es el mismo para todas las empresas. Para considerar la presencia de diferencias en las ordenadas al origen se estima el modelo de efectos fijos (EF), el cual incluye variables dummies que captan efectos específicos en las empresas. Para comprobar su significancia estadística se realiza una prueba F, cuya hipótesis nula es que los coeficientes de las mismas son iguales a cero. Por último, se estima el modelo de efectos aleatorios (EA), en donde el término de perturbación es, en el cual es un término de error aleatorio no observable que representa el componente de error específico individual y es el error combinado de la serie de tiempo con el corte transversal. Un supuesto es que no está correlacionado con las variables explicativas, es decir, .

Se realiza la prueba de Breusch y Pagan Multiplicador de Lagrange (BPLM) (1979) para probar la presencia de efectos aleatorios. La hipótesis nula es que la varianza individual específica es cero, es decir, . En el presente análisis, el test BPLM rechaza la hipótesis nula, es decir, se acepta la presencia de efectos aleatorios en el modelo. Esto se confirma con la aplicación de la prueba de Hausman (1978).

Los resultados de las estimaciones se presentan en la tabla 3 , y muestran que mayores niveles de endeudamiento se asocian con un aumento en la razón de fragilidad financiera. Tanto la razón de la deuda de corto plazo/patrimonio neto (DCP) como la razón de deuda de largo plazo/patrimonio neto (DLP) resultaron estadísticamente significativas al 99\%. Por cada incremento de 1 unidad en su razón DCP, las empresas disminuirán 1,26 unidades su IRC. Asimismo, a mayor deuda de largo plazo, las empresas también tienen menor capacidad de cubrir sus compromisos financieros. Por cada unidad de incremento en la DLP, el IRC se reduce en 2,32 unidades.

El índice de los términos de intercambios medido en dólares estadounidenses (Itdlls) también fue estadísticamente significativa al 99\%, y con el signo positivo esperado. Eso significa que una mejora en los precios de las exportaciones con relación al precio de las importaciones reduce la fragilidad financiera, en tanto aumenta los ingresos de las grandes empresas manufactureras relacionados a las exportaciones. Un alza de 1 unidad en el Itidlls genera un incremento de 0,33 sobre el IRC.

La tasa de interés de la economía mexicana (rMEX) es otra variable estadísticamente significativa (al 90\%) y que cumple con el signo esperado. Aumentos en la tasa de interés implican un aumento de los compromisos financieros, y en consecuencia un deterioro de la capacidad de las empresas para cubrir sus compromisos financieros. En particular, un aumento de $1 \%$ en la tasa de interés se asocia a un decremento de 0,000174 en el índice IRC. 
Tabla 2. Resultados de las estimaciones econométricas

\begin{tabular}{|c|c|c|c|}
\hline IRC & $\mathrm{MCO}$ & FE & $\mathrm{RE}$ \\
\hline \multirow[t]{2}{*}{ rMex } & -0.0000181 & -0.000017 & -0.000174 \\
\hline & $(0.078)^{* *}$ & $(0.07)^{* *}$ & $(0.065)^{* *}$ \\
\hline \multirow[t]{2}{*}{ Itidlls } & 0.3550286 & 0.317638 & 0.3303684 \\
\hline & $(0.022)^{* * *}$ & $(0.025)^{* * *}$ & $(0.020)^{* * *}$ \\
\hline \multirow[t]{2}{*}{$X$} & $8.43 \mathrm{E}-06$ & 0.00000851 & 0.00000848 \\
\hline & $(0.149)^{*}$ & $(0.111)^{*}$ & $(0.113)^{*}$ \\
\hline \multirow[t]{2}{*}{$\mathrm{CG}$} & -13.05653 & -11.528856 & -12.04876 \\
\hline & $(0.025) * * *$ & $(0.030) * * *$ & $(0.024)^{* * *}$ \\
\hline \multirow[t]{2}{*}{$\mathrm{DCP}$} & -1.62896 & -1.071123 & -1.260526 \\
\hline & $(0.000)^{* * *}$ & $(0.010) * * *$ & $(0.002)^{* * *}$ \\
\hline \multirow[t]{2}{*}{ DLP } & $-3.11 \mathrm{E}+00$ & -1.9040002 & $-2.32 \mathrm{E}+00$ \\
\hline & $(0.000)^{* * *}$ & $(0.005)^{* * *}$ & $(0.000)^{* * *}$ \\
\hline \multirow[t]{2}{*}{ CONS } & $-1.11 \mathrm{E}+01$ & $-1.02 \mathrm{E}+01$ & $-1.05 \mathrm{E}+01$ \\
\hline & $(0.122)^{*}$ & $(0.120)^{*}$ & $(0.112)^{*}$ \\
\hline \multirow[t]{2}{*}{ Prueba F } & 6.35 & 2.89 & \\
\hline & $(0.0000)^{* * *}$ & $(0.0100)^{* * *}$ & \\
\hline \multirow[t]{2}{*}{ Breush-Pagan } & & & 23.86 \\
\hline & & & $(0.0006)^{* * *}$ \\
\hline \multirow[t]{2}{*}{ Hausman } & & 7.04 & \\
\hline & & $(0.1336)^{*}$ & $(0.1336)^{*}$ \\
\hline
\end{tabular}

Nota: $99 \%=* * * 95 \%=* * * 90 \%$ y menos $=*$

Fuente: elaboración propia con base en datos de Economatica y Stata 12.

La tasa de crecimiento anual del consumo público también resultó estadísticamente significativa al 99\%. Sin embargo, no tuvo el signo esperado. Además, llama la atención de la magnitud de su coeficiente, que indicaría que un aumento del $1 \%$ en esta variable se asocia a una disminución 12,04 en el IRC. No obstante, 
se requieren estimaciones adicionales para determinar la presencia de una causalidad inversa que asocie el uso del consumo público como medida contracíclica en períodos de fragilidad financiera, derivados de la caída en la actividad económica.

Por su parte, las exportaciones hacia la economía de Estados Unidos (X) cumplen con el signo esperado, aunque no resultaron estadísticamente significativas.

Es importante destacar que la presencia de la deuda de corto y la deuda de largo plazo como variables significativas es un resultado consistente en las tres especificaciones estimadas. Es decir, el endeudamiento es el principal determinante de la fragilidad financiera, la cual se profundizó con el cambio de condiciones en los mercados internacionales.

Por último, es necesario mencionar que también se estimó la significancia estadística de variables como la tasa de interés de los fondos federales, el consumo privado, las remesas, la inversión extrajera directa, y el crecimiento promedio anual de las exportaciones, pero no resultaron estadísticamente significativas.

\section{CONCLUSIONES}

La hipótesis de la inestabilidad financiera de Minsky es un planteamiento que destaca la inherente inestabilidad del sistema económico, y cómo la estructura financiera se vuelve determinante de una mayor o menor fragilidad.

México no fue el origen de la crisis financiera subprime pero sus efectos se transmitieron a las grandes empresas por los estrechos vínculos entre ambas economías. El análisis de la evolución de la estructura financiera de las grandes empresas manufactureras mexicanas con el marco analítico de Minsky demuestra la pertinencia de este último y de las propuestas para su evaluación empírica.

Se observó que la estructura financiera era frágil incluso antes de la crisis subprime, y que ello se asoció a los procesos de inversión financiados a través de endeudamiento, por lo que el surgimiento de la crisis profundizó su grado de fragilidad. A raíz de esta última, las condiciones de financiamiento se volvieron adversas, en particular por el aumento de la tasa de interés, a lo que se sumó la reducción de sus ingresos no financieros, dada su alta dependencia del mercado estadounidense. En términos de Minsky, la caída en la actividad de la economía estadounidense supuso una mayor fragilidad financiera en las empresas derivada de que los flujos de efectivo no validaron inversiones de períodos precedentes, $y$ en consecuencia 
provocaron el estancamiento de los procesos de inversión, así como del empleo y el ingreso asociados.

Los resultados de la estimación econométrica de datos en panel confirman lo anterior. Se observa que las variables de mayor incidencia en el indicador de fragilidad financiera fueron las deudas de corto y largo plazo, así como los términos de intercambio.

En el período posterior a la crisis subprime las estructuras financieras de varias empresas se recuperaron a los niveles previos a la crisis, pero otras no han podido hacerlo, por lo que siguen resintiendo los impactos de esta crisis. Esto también se explica por el entorno de bajo crecimiento general de la economía mexicana en las dos últimas décadas, en promedio, de alrededor del $2 \%$ anual.

En este contexto, y ante el hecho de que las crisis no se pueden evitar, como advierte Minsky, las grandes empresas mexicanas del sector manufacturero deben buscar alternativas para incrementar su actividad y fortalecer su estructura financiera. Una primera vía es continuar con la expansión y diversificación de sus mercados y fuentes ingresos. La segunda opción, y que es complementaria a la primera, consiste en fortalecer el mercado interno. No obstante, esta segunda opción requiere de la participación gubernamental para impulsar una política industrial que genere articulaciones entre las empresas del sector productivo doméstico, en especial con las pequeñas y medianas empresas.

Adicionalmente, se requiere tomar en cuenta las propuestas de crear políticas específicas con el propósito de atenuar los impactos de los ciclos económicos (Agosin y Huaita, 2011). En relación con este aspecto en particular, adquiere relevancia el estudio del papel que pueden desempeñar las políticas gubernamentales contracíclicas y las instituciones financieras públicas como el propio banco central y los bancos de desarrollo no solo nacionales sino de carácter regional que contribuyan hacia la creación de estructuras financieras cubiertas, como proponía Minsky.

\section{BIBLIOGRAFÍA}

Agosin, M., \& Huaita, F. (2011). Capital flows to emerging economies: Minsky in the tropics, Cambridge Journal of Economics, 35(4), 663-683.

Argitis, G., \& Nikolaidi M. (2014).The financial fragility and the crisis of the Greek government sector, International Review of Applied Economics, 28(3), 274292, doi: 10.1080/02692171.2013.858667 
Banco de México (2010). Informe anual. Recuperado de http://www.banxico. org.mx/publicaciones-y-discursos/publicaciones/informes-periodicos/ anual/\%7B4DD504A9-510A-F0DC-76B2-D028BE9FB374\%7D.pdf

Beshenov, S., \& Rozmainsky I. (2015). Hyman Minsky's instability hypotesis and the greek debt crisis. Rusian Journal of Economics, 1(4), 419-438.

Breusch, T. S, \& Pagan, A. R. (1979). Simple test for heteroscedasticity and random coefficient variation, Econometrica (The Econometric Society), 47(5), 12871294.

Cuevas, V. (2013). La crisis hipotecaria subprime y sus efectos sobre México. Análisis Económico, 28(67), 124-151

Damodaran, A. (2011). Applied corporate finance. Hoboken, New Jersey: John Wiley \& Sons.

Garrido, C., \& Ortiz, C. (2009). De crisis en crisis: La evolución reciente de las grandes empresas mexicanas. Problemas del desarrollo, 40(1569), 48-75.

Garrido, C. (2005). Desarrollo económico y procesos de financiamiento en México. Transformaciones contemporáneas y dilemas actuales ( $1 .{ }^{a}$ ed.). México: Siglo XXI.

Chapoy, A., \& Girón, A. (2009). Financiarización y titulización: un momento Minsky. Economía UNAM, 6(16), 44-56.

Hausman, J. A. (1978) Specifications tests in econometrics, Econometrica, 46(6), 1251-1271.

INEGI. (2015). Censos Económicos 2014. Resultados definitivos. Recuperado de http://internet.contenidos.inegi.org.mx/contenidos/productos/prod_serv/ contenidos/espanol/bvinegi/productos/nueva_estruc/promo/pprd_ce2014.pdf

Kregel, J. (2008). Minsky's cushions of safety: Systemic risk and the crisis in the US subprime mortgage market, (Report Public Policy Brief, No. 93). Recuperado de http://www.levy.org/pubs/ppb_93.pdf

Mántey G., \& Quintana L. (2013). Política monetaria en mercados bancarios imperfectos, abiertos y desregulados: un análisis post-keynesiano En $\mathrm{N}$. Levy \& T. López (Comp.). Financiarización y modelo de acumulación: aportes desde los países en desarrollo (9-32). México: Facultad de Economía, UNAM.

Mulligan, R., Lirely, R., \& Coffee D. (2014). An empirical Examination of Minsky's financial Hypothesis: From Market process to Austrian Business Cycle. Journal des Economistes et des Etudes Humaines, De Gruyter, 20(1), 1-17.

Minsky, H. (1986). Stabilizing an unstable economy. A Twentieth Century Fund Report. Estados Unidos: Yale University Press.

Palley, T. (2012). The Rise and Fall of Export-led Growth. Investigación Económica, 70(276), 125-162.

Stockhammer, E. (2004). Financialisation and the slowdown of accumulation. Cambridge Journal of Economics, 28(5), 719-741.

Teplova, T. V. (2011). Investment. Rusia: Publishing house Yurait. 
(C) 2019 por los autores; licencia otorgada a la Revista Estudios Económicos. Este artículo es de acceso abierto y distribuido bajo los términos y condiciones de una licencia Atribución-No Comercial 3.0 Unported (CC BY-NC 3.0) de Creative Commons. Para ver una copia de esta licencia, visite http://creativecommons.org/ licenses/by-nc/3.0/ 